Natural Hazards and Earth System Sciences, 5, 555-559, 2005

SRef-ID: 1684-9981/nhess/2005-5-555

European Geosciences Union

(C) 2005 Author(s). This work is licensed

under a Creative Commons License.

\title{
Multifractality in local geomagnetic field at Etna volcano, Sicily (southern Italy)
}

\author{
G. Currenti ${ }^{1}$, C. del Negro ${ }^{1}$, V. Lapenna ${ }^{2}$, and L. Telesca ${ }^{2}$ \\ ${ }^{1}$ Istituto Nazionale di Geofisica e Vulcanologia, Sezione di Catania, Catania, Italy \\ ${ }^{2}$ Instituto di Metodologie per l'Analisi Ambientale,CNR, C. da S. Loja, 85050 Tito (PZ), Italy \\ Received: 15 June 2005 - Revised: 11 July 2005 - Accepted: 11 July 2005 - Published: 29 July 2005 \\ Part of Special Issue "Seismic hazard evaluation, precursory phenomena and seismo electromagnetics"
}

\begin{abstract}
We applied the Multifractal Detrended Fluctuation Analysis (MF-DFA), which allows to detect multifractality in nonstationary signals, to the hourly means of local geomagnetic field recorded at Mt. Etna volcano (southern Italy). We studied the signal measured at one geomagnetic station, installed at the summit of volcano, which was characterized by a strong eruption on 27 October 2002. We analyzed two frames of signals, one measured before the eruption and the other after, in order to evaluate dynamical changes induced by the eruptive event. Our findings show that: i) the geomagnetic time series is multifractal; ii) the multifractal degree of the signal decreases after the occurrence of eruption. This study aims to propose another approach to investigate the complex dynamics of volcano-related geomagnetic field.
\end{abstract}

\section{Introduction}

Volcanoes are complex dynamical systems controlled by interactions of many processes, of whom the most spectacular effects have been observed near eruptive events. There are many uncertainties in the controlling parameters. However, intensive monitoring of recent eruptions of Kilauea, Mount Etna, Mount Unzen, and Montserrat has generated integrated time series of data, which have advanced understanding of volcanic processes. Contemporary methodologies, able to detect timescale structures in observational time series, have been particularly useful to obtain information on the features and on the causes of variation at the different timescales. In particular, time-scaling analysis techniques, developed to draw qualitative and quantitative information from time series, have been applied recently to the study of a large variety of irregular, erratic signals and by now have proved to be very valuable to detect deep dynamical features.

Correspondence to: L. Telesca

(ltelesca@imaa.cnr.it)
At Mount Etna it has already been found that the dynamics of volcano is characterized by scaling respect to several features. In particular the sequence of eruptive events shows time-scaling over a relatively wide range of timescales, pointing to the presence of correlated structures in the point process, which models the sequence of eruptions (Telesca et al., 2002). Also the seismicity of Mt. Etna shows timescaling behavior. Vinciguerra and Barbano (2000), analyzing the box-counting time fractal dimension of the seismicity, found a non random distribution over time of the earthquakes. Furthermore, variation of the time-fractal dimension was detected at different timescales. Variations from short to mid term scales (order of days) in correspondence to the onset of eruptions (in which sharp decrease of the dimension was followed by sharp increase) can be related to rock fracturing processes induced by the penetration of magma into shallow layers, or by fracture systems opening linked to early stages of the eruption. The deep earthquakes show approximately two time-fractal trends: sharp variation of the clustering at the onset of the eruption, probably due to overall volcano edifice responding to the final phases of the magma rise; the other is a relative lowering of the fractal dimension during and between the eruptive cycle (order of years).

In the recent past the magnetic monitoring at Mt. Etna has proved its ability to detect significant time variations in the local magnetic field linked to volcanic activity (Del Negro et al., 1997; Del Negro et al., 1998). Detection of magnetic anomalies has often been proposed for monitoring the modifications in the stress field or the thermodynamic state within the volcanic edifice and providing a tool to forecast eruptions.

Magnetic anomalies attributable to the dynamics of volcano show several temporal scales, which reflect the superposition of different processes. At least variations at three different timescales can be considered. Longterm trend (months to years) are probably associated with 


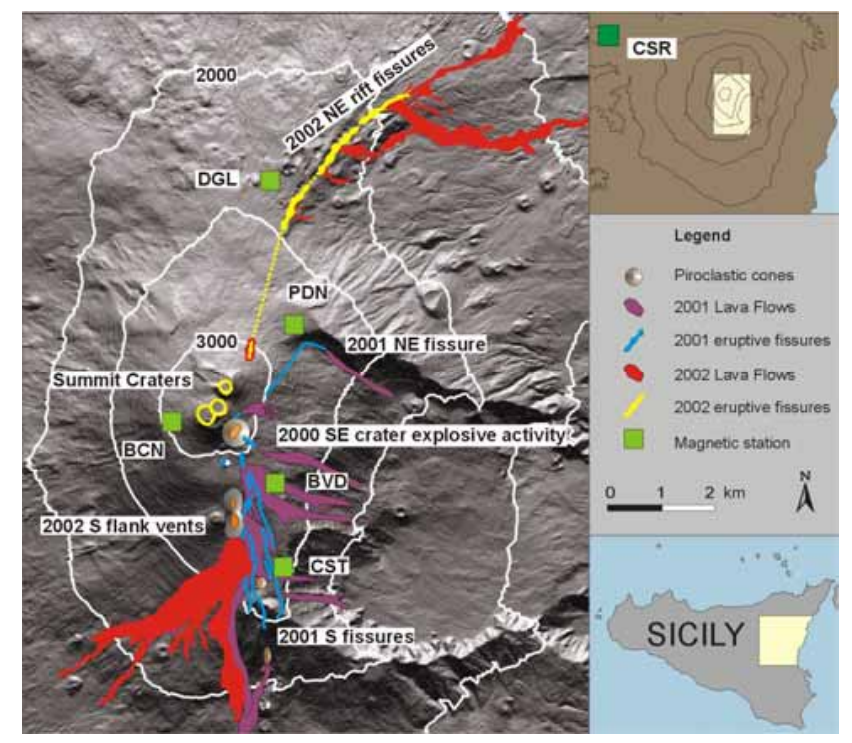

Fig. 1. Schematic map showing the area covered by the lava flows from 2001 and 2002 Etna eruptions. Locations of magnetic stations are shown as well.

demagnetization/remagnetization of rocks due to thermal process inside the volcano. The amount of these variations can reach several tens nanoteslas depending on the rocks magnetization (Yukutake et al., 1990). Mid-term trend signals (weeks to months), with amplitude of about few nanoteslas, include near-surface thermal demagnetization effects, stress field induced changes, and effects from rotation/displacement of magnetized material (Zlotnicki et al., 1993). Short-term variations (seconds to days) could result from instantaneous variations of rocks magnetization induced by local stress redistribution and from fluid flow current through fissures within the volcanic edifice accompanying fault ruptures and fracture opening (Mueller and Johnston, 1998; Sasai et al., 2002). Thus, the characterization of the volcanomagnetic signals, which seem to depend on the structural heterogeneity and the dynamics of the plumbing system of each volcanic edifice, can be a useful instrument both for improving the monitoring of active volcanoes as well as developing a greater understanding of the preeruptive mechanisms which produce them.

To quantitatively characterize geomagnetic dynamics, we need to use robust methodologies in order to extract features hidden in their complex fluctuations. Fractality is one of the features of such complexity. Fractal processes are characterized by scaling behaviour, which leads naturally to power-law statistics (Thurner et al., 1997 and references therein). Several methods can be applied to reveal the fractality of a signal, such as the most standard technique of the power spectral density, or the more robust Higuchi method (Higuchi, 1988; 1990), or the Detrended Fluctuation Analysis (Peng et al., 1995), which allows the detecting of scaling behaviours in experimental time series, very often affected by trends and nonstationarities, which cause spurious detection of correlations.
But all these techniques are monofractal, and not sufficient to describe the overall scaling behaviour of a signal, which could be multifractal.

What does multifractality mean? A multifractal is an object which needs many exponents to characterize its scaling properties. It can be decomposed into many sub-sets characterized by different scaling exponents. Thus multifractals are intrinsically more complex and inhomogeneous than monofractals, and characterize systems featured by irregular dynamics, with sudden bursts of high frequency fluctuations.

The aim of the present paper is the dynamical investigation of a geomagnetic field at Mt. Etna volcano in southern Italy, in order to characterize the multifractality of such time series and reveal dynamical change induced by eruptions.

\section{Data}

Detection of clear magnetic signals associated with the renewal of the volcanic activity led to an intensification of the magnetic monitoring of Mt. Etna. Since the end of 1998 a permanent magnetic network equipped with Overhauser effect magnetometers has been set up (Del Negro et al., 2002). The present network consists of five stations placed on the volcanic edifice (CST, BVD, BCN, PDN, and DGL), flanked by a sixth external reference station (CSR) installed further west (about $50 \mathrm{~km}$ ) on the Nebrodi Mountains (Fig. 1). Stations on Etna are located at elevations ranging between 2350 and $3000 \mathrm{~m}$ above sea level along a North-South profile crossing the summit craters. This layout is symmetrical with respect to the central craters and should allow continuous prospecting of the geomagnetic field along this section of the volcano. The sites were carefully tested before the stations were installed and the inspection revealed the presence of low magnetic gradient (less than $50 \mathrm{nT} / \mathrm{m}$ ) and low background noise level. To avoid large effects due to strong local static magnetic anomalies, the sensor is fixed at the top of a $4 \mathrm{~m}$ high pole. All sites sample synchronously every $10 \mathrm{~s}$ and transmit data via mobile phone to the INGV-Catania Section where these data are processed and analyzed.

In the present paper we analyze the hourly time-variability of the geomagnetic signals measured at PDN site two months before and two after the strong eruption occurrring on $27 \mathrm{Oc}-$ tober 2002 at Mt. Etna volcano. The geomagnetic field variations attributable to the dynamics of volcano can be useful indicators of the modifications of the stress field or of the thermodynamic state within the volcanic edifice. However, the volcanic geomagnetic field varies with amplitudes of $1 \sim 10 \mathrm{nT}$ in most cases, while changes up to a few hundreds nT are caused by ionospheric and magnetospheric currents, and secular variations. Therefore, it is necessary to remove these variations in order to highlight an on-going physical process inside a volcano. Simple differences in the total intensity with respect to the simultaneous value at the CSR remote reference (located at a magnetically quiet site) are used to reduce secular variations and external geomagnetic disturbances (these contributions can be considered spatially 
uniform on the scale of the volcano). In Fig. 2a the total intensity variations observed at PDN relative to CSR are shown; there are some gaps due to equipment drawbacks. Nevertheless, very much larger time variations, with periods from minutes to years, are present in these differences. For example, also in the absence of any volcanic activity, some of the time changes can occur because of secondary fields induced in the crustal rocks by external current systems. At different positions this disturbance appears with different properties and characteristics because of its interaction with the local environment (Davis et al., 1981), but it should be predictable, because the variations at the different stations are correlated by their electromagnetic impedances. Therefore, a predictive filtering could be used to further reduce the changes in the difference fields due to contrasting responses at magnetometer sites, with the filters giving the relative responses between sites. The predictive filtering estimates the variations that are common to different sites, and the filter output is a prediction of the signal based on observations in other sites. The residual field (the difference between the observed values and the predicted values) contains only the effects that are spatially local. This one represents the signal to be analyzed. It is worth noting that total field differences are also dependent on the direction of the disturbing field (Davis et al., 1981). The directional effect has meant that vector information as well as that from total fields must be used as input to the filter to take this into account. In our case, the vector magnetic field data from L'Aquila Geomagnetic Observatory, which is the closest vector magnetometer, are utilized. Because of the nonstationarities of the geomagnetic signal, we propose an adaptive filter which estimates and updates the filter parameters continuously by means of the new observations, so that predictions closely match the observed data. In this case, the three component fields of the vector magnetometer at L'Aquila $(X, Y, Z)$ and the total field at CSR reference station (of Etna array) represent inputs to the adaptive filter, and the estimated signal $y(t)$ at PDN is the output, as reported below:

$$
\begin{aligned}
\hat{y}(t) & =\sum_{i=0}^{m_{1}} a_{1 i}(t) X(t-i)+\sum_{i=0}^{m_{2}} a_{2 i}(t) Y(t-i) \\
& +\sum_{i=0}^{m_{3}} a_{3 i}(t) Z(t-i)+\sum_{i=0}^{m_{4}} a_{4 i}(t) C S R(t-i)
\end{aligned}
$$

where $a_{i j}$ are the time-varying parameters, $n$ and $m_{1}, \ldots \mathrm{m}_{4}$ predictors.

The adaptive filter is effective enough and the changes in October 2002 are the only significant ones observed during the analyzed period (Fig. 2b). After differential magnetic field measurements were filtered from the external noise by using adaptive filters, we recognized two stages in the total intensity changes, which are closely related to different volcanic events: (a) a sudden drop of about $4-5 \mathrm{nT}$ associated with 26 October seismic swarm recorded beneath the summit craters; and (b) step-like variations of 9-10 nT coincident with 27 October eruptive fissures opening up in the

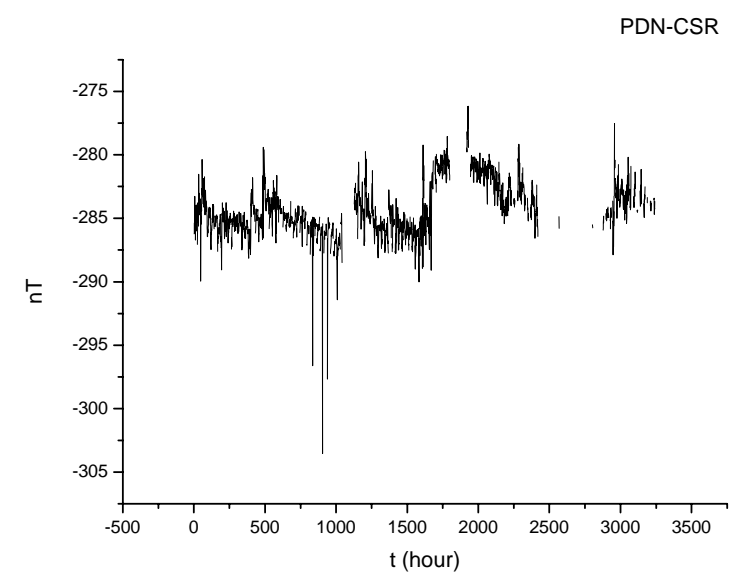

a)

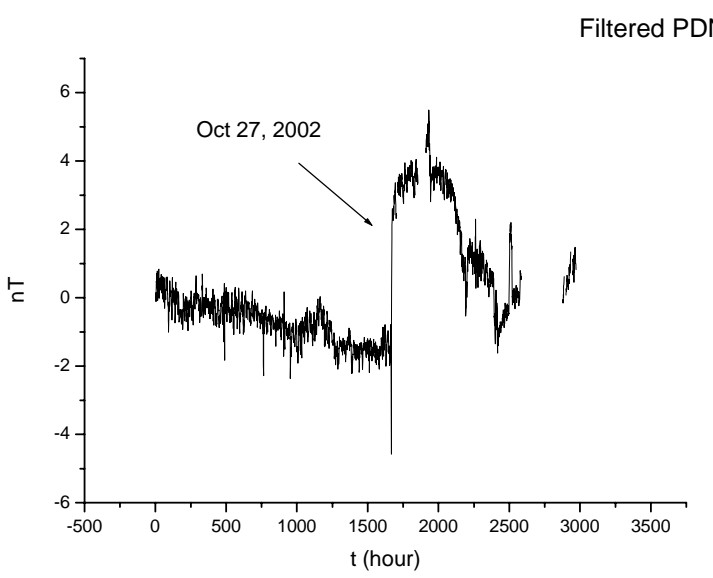

b)

Fig. 2. Time variation of the geomagnetic signal measured at PDN station referred to CSR (a) and after removal of external effects by adaptive filtering (b); the arrow indicates the time of occurrence of the strong eruption on 27 October 2002

north flank (Del Negro et al., 2004). Until 26 October no significant changes were observed at PDN magnetic station. Therefore, we split the signal into two frames, before and after the 2002 eruption, two months long each. We analyzed the multifractal properties of both frames, in order to evidence possible dynamical changes, induced by the eruption.

\section{Methods and data analysis}

Observational data often present clear irregular dynamics, characterized by sudden bursts of high frequency fluctuations, which suggest performing a multifractal analysis evidencing the presence of different scaling behaviours for different intensities of fluctuations. Furthermore, the signal may appear nonstationary.

The Multifractal Detrended Fluctuation Analysis (MFDFA) (Kantelhardt et al., 2002) is a useful tool to characterize multifractality in nonstationary data. 


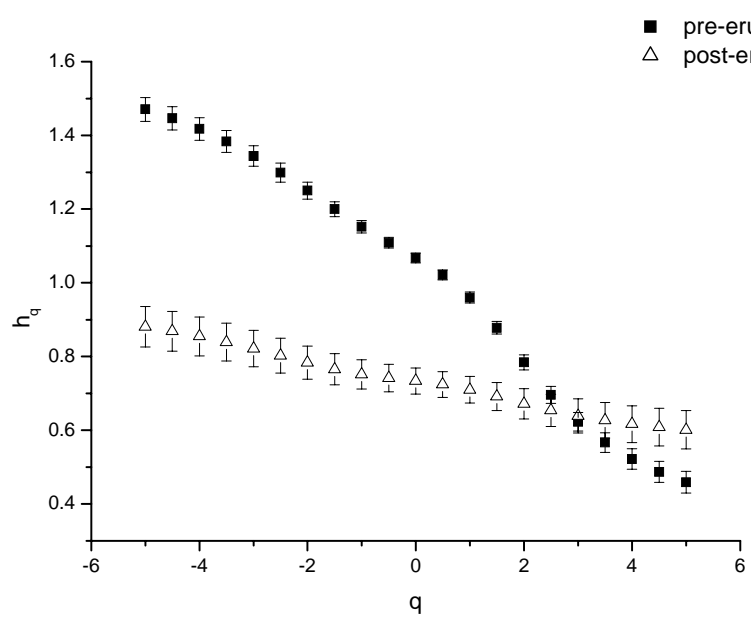

Fig. 3. $\mathrm{h}(\mathrm{q}) \sim \mathrm{q}$ relation for the geomagnetic PDN time series.

The method is based on the conventional detrended fluctuation analysis (Peng et al., 1995). It operates on the time series $x(i)$, where $i=1,2, \ldots, N$ and $N$ is the length of the series. With $\mathrm{x}_{\text {ave }}$ the mean value is indicated. Assume that $\mathrm{x}(\mathrm{i})$ are increments of a random walk process around the average $\mathrm{x}_{\text {ave }}$, the "trajectory" or "profile" is given by the integration of the signal

$y(i)=\sum_{k=1}^{i}\left[x(k)-x_{\mathrm{ave}}\right]$

Next, the integrated time series is divided into $\mathrm{N}_{S}=\mathrm{int}(\mathrm{N} / \mathrm{s})$ no overlapping segments of equal length s. Since the length $\mathrm{N}$ of the series is often not a multiple of the considered time scale s, a short part at the end of the profile y(i) may remain. In order not to disregard this part of the series, the same procedure is repeated starting from the opposite end. Thereby, $2 \mathrm{~N}_{S}$ segments are obtained altogether. Then the local trend for each of the $2 \mathrm{~N}_{S}$ segments is calculated by a least square fit of the series. Then one calculates the variance

$F^{2}(s, v)=\frac{1}{s} \sum_{i=1}^{s}\left\{y[(v-1) s+i]-y_{v}(i)\right\}^{2}$

for each segment $v, \nu=1, . ., \mathrm{N}_{S}$ and

$F^{2}(s, v)=\frac{1}{s} \sum_{i=1}^{s}\left\{y\left[N-\left(v-N_{S}\right) s+i\right]-y_{v}(i)\right\}^{2}$

for $\nu=\mathrm{N}_{S}+1, \ldots, 2 \mathrm{~N}_{S}$. Here, $\mathrm{y}_{v}(\mathrm{i})$ is the fitting line in segment $v$. Then, an average over all segments is performed to obtain the q-th order fluctuation function

$F_{q}(s)=\left\{\frac{1}{2 N_{S}} \sum_{\nu=1}^{2 N_{S}}\left[F^{2}(s, v)\right]^{\frac{q}{2}}\right\}^{\frac{1}{q}}$

where, in general, the index variable q can take any real value except zero.

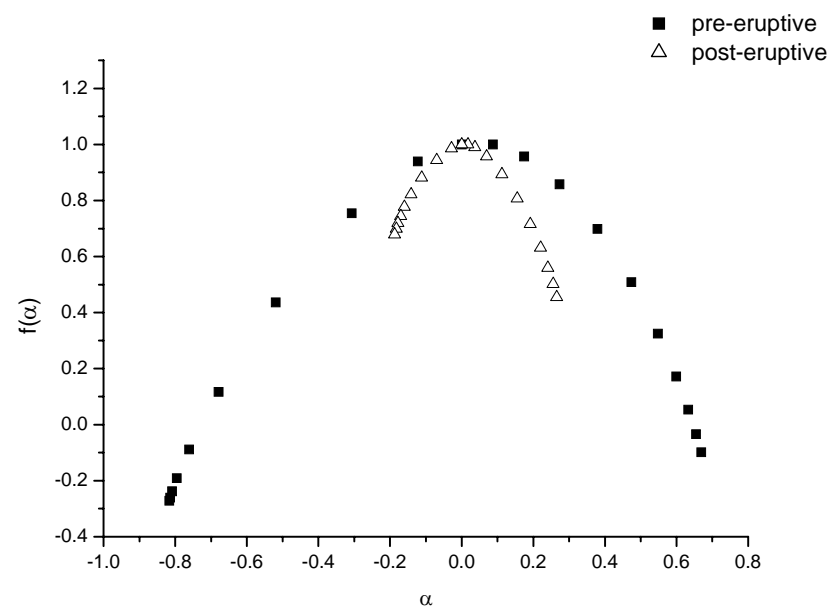

Fig. 4. Singularity spectra of the geomagnetic PDN time series.

Repeating the procedure described above, for several time scales $\mathrm{s}, \mathrm{F}_{q}(\mathrm{~s})$ will increase with increasing $\mathrm{s}$. Then analyzing $\log$-log plots $\mathrm{F}_{q}(\mathrm{~s})$ versus $\mathrm{s}$ for each value of $\mathrm{q}$, the scaling behaviour of the fluctuation functions can be determined. If the series $\mathrm{x}_{i}$ is long-range power-law correlated, $\mathrm{F}_{q}(\mathrm{~s})$ increases for large values of $\mathrm{s}$ as a power-law

$F_{q}(s) \propto s^{h(q)}$.

The value $\mathrm{h}(0)$ corresponds to the limit $\mathrm{h}(\mathrm{q})$ for $\mathrm{q} \rightarrow 0$, and cannot be determined directly using the averaging procedure of Eq. (5) because of the diverging exponent. Instead, a logarithmic averaging procedure has to be employed,

$F_{0}(s) \equiv \exp \left\{\frac{1}{4 N_{S}} \sum_{\nu=1}^{2 N_{S}} \ln \left[F^{2}(s, v)\right]\right\} \approx s^{h(0)}$.

In general the exponent $\mathrm{h}(\mathrm{q})$ will depend on $\mathrm{q}$. In particular for monofractal series it is independent of q. For stationary time series, $\mathrm{h}(\mathrm{q})$ is the well-defined Hurts exponent $\mathrm{H}$ (Feder, 1988). Thus, we call $\mathrm{h}(\mathrm{q})$ the generalized Hurst exponent. The different scaling of small and large fluctuations will yield a significant dependence of $\mathrm{h}(\mathrm{q})$ on $\mathrm{q}$. For positive $\mathrm{q}$, the segments $v$ with large variance (i.e. large deviation from the corresponding fit) will dominate the average $\mathrm{F}_{q}(\mathrm{~s})$. Therefore, if $\mathrm{q}$ is positive, $\mathrm{h}(\mathrm{q})$ describes the scaling behaviour of the segments with large fluctuations; and generally, large fluctuations are characterized by a smaller scaling exponent $\mathrm{h}(\mathrm{q})$ for multifractal time series. For negative $\mathrm{q}$, the segments $v$ with small variance will dominate the average $\mathrm{F}_{q}(\mathrm{~s})$. Thus, for negative $\mathrm{q}$ values, the scaling exponent $\mathrm{h}(\mathrm{q})$ describes the scaling behaviour of segments with small fluctuations, usually characterized by larger scaling exponents.

Figure 3 shows the q-dependence of the generalized Hurst exponent $\mathrm{h}$ (q) determined by fits in the regime $10 \mathrm{~h}<\mathrm{s}<\mathrm{N} / 4$, where $\mathrm{N}$ indicates the length of the series and for $\mathrm{q}$ ranging between -5 and 5 with 0.5 step. In the plot the $\mathrm{h}(\mathrm{q}) \sim \mathrm{q}$ relation is represented for both the signal frames. We observe 
that before the eruption the signal is characterized by a variability of the generalized Hurst exponents larger than after the eruption.

The multifractal scaling exponents $\mathrm{h}(\mathrm{q})$ are directly related to the scaling exponents $\tau(\mathrm{q})$ defined by the standard partition function multifractal formalism (Kantelhardt et al., 2002)

$\tau(q)=q h(q)-1$.

The singularity spectrum $f(\alpha)$ is related to $\tau(q)$ by means of the Legendre transform (Parisi and Frish, 1985),

$\alpha=\frac{d \tau}{d q}$

$f(\alpha)=q \alpha-\tau(q)$,

where $\alpha$ is the Hölder exponent and $\mathrm{f}(\alpha)$ indicates the dimension of the subset of the series that is characterized by $\alpha$. The singularity spectrum quantifies in details the longrange correlation properties of a time series. Figure 4 shows the multifractal spectrum $\mathrm{f}(\alpha)$ for both the signal frames; the multifractal spectrum of the signal frame before the eruption is much larger than that after the event.

\section{Conclusions}

The geophysical phenomenon underlying the geomagnetic activity connected to volcanic dynamics is rather complex. The multifractal analysis could drive a better understanding of such complexity. The MF-DFA, by means of the calculation of the generalized Hurst exponents and the singularity spectra, has revealed that the volcano-magnetic signal is multifractal. But, the occurrence of an eruptive event changes such multifractality, which is reduced after the eruption. The decrease of the multifractal degree (measured by the range of the Hurst exponents or, equivalently, by the width of the multifractal spectrum) after the eruption indicates a loss of heterogeneity. The corresponding contraction of the singularity spectrum indeed means a transition from heterogeneous to homogeneous patterns (Ida et al., 2005).

Thus, the characterization of the volcanomagnetic signals, which seem to depend on the structural heterogeneity and the dynamics of the plumbing system of each volcanic edifice, can be a useful instrument both for improving the monitoring of active volcanoes as well as developing a greater understanding of the pre-eruptive mechanisms which produce them.

Edited by: P. F. Biagi

Reviewed by: two referees

\section{References}

Davis, P. M., Jackson, D. D., Searls, C. A., and McPhernon, R. L.: Detection of tectonomagnetic events using multichannel predictive filtering, J. Geophys. Res., 86, 1731-1737, 1981.

Del Negro, C., Ferrucci, F., and Napoli, R.: Retrieval of large volcanomagnetic effects observed during the 1981 eruption of Mt. Etna, Ann. Geophys., XL, 547-562, 1997.

Del Negro, C. and Ferrucci, F.: Magnetic history of a dyke on Mt. Etna, Geophys. J. Int., 133, 451-458, 1998.

Del Negro, C., Napoli, R., and Sicali, A.: Automated System for Magnetic Monitoring of Active Volcanoes, Bull. Volcanol., 64, 94-99, 2002.

Del Negro C., Currenti G., Napoli R., and Vicari A.: Volcanomagnetic changes accompanying the Onset of the 2002-2003 Eruption of Mt. Etna (Italy), Earth and Planet. Sci. Lett., 229, 1-14, 2004.

Ida, Y., Hayakawa, M., Adalev, A., and Gotoh, K.: Multifractal analysis for the ULF geomagnetic data during the 1993 Guam earthquake, Nonlin. Processes Geophys., 12, 157-162, 2005, SRef-ID: 1607-7946/npg/2005-12-157.

Feder, J.: Fractals (Plenum Press, New York), 1988.

Higuchi T.: Approach to an irregular time series on the basis of the fractal theory, Physica D, 31, 277-283, 1988.

Higuchi T.: Relationship between the fractal dimension and the power law index for a time series: a numerical investigation, Physica D, 46, 254-264, 1990.

Kantelhardt J. W., Zschiegner S. A., Konscienly-Bunde E., Havlin S., Bunde A., and Stanley H. E.: Multifractal detrended fluctuation analysis of nonstationary time series, Physica A, 316, 87114, 2002.

Mueller, R. J. and Johnston, M. J. S.: Review of magnetic field monitoring near active faults and volcanic calderas in California: 1974-1995, Phys. Earth Planet. Int., 105, 131-144, 1998.

Parisi G. and Frisch U.: In Turbulence and Predictability in Geophysical Fluid Dynamics and Climate Dynamics, (Eds.) Ghil, M., Benzi, R., and Parisi, G. (North Holland, Amsterdam), 1985.

Peng C.-K., Havlin S., Stanley H. E., and Goldberger A. L.: Quantification of scaling exponents and crossover phenomena in nonstationary heartbeat time series, CHAOS, 5, 82-87, 1995.

Sasai, Y., Uyeshima, M., Zlotnicki, J., Utada, H., Kagiyama, T., Hashimoto, T., and Takahashi, Y.: Magnetic and electric field observations during the 2000 activity of Miyake-jima volcano, Central Japan, Earth Planet. Sci. Lett., 203, 769-777, 2002.

Telesca, L., Cuomo, V., Lapenna, V., and Macchiato, M.: Timeclustering analysis of volcanic occurrence sequences, Phys. Earth Planet. Int., 131, 47-62, 2002.

Thurner S., Lowen S. B., Feurstein M. C., Heneghan C., Feichtinger H. C., and Teich M. C.: Analysis, Synthesis, and Estimation of Fractal-Rate Stochastic Point Processes, Fractals, 5, 565-596, 1997.

Vinciguerra, S. and Barbano, M. S.: Clustering of Seismicity at Mt. Etna, Phys. Chem. Earth, 25, 699-703, 2000.

Yukutake, T., Utada, H., Yoshino, T., Watanabe, H., Hamano, Y., Sasai, Y., Kimoto, E., Otani, K., and Shimomura, T.: Changes in the geomagnetic total intensity observed before the eruption of Oshima Volcano in 1986, J. Geomag. Geoelectr., 42, 277-290, 1990.

Zlotnicki, J., Le Mouel, J. L., Delmond, J. C., Pambrun, C., and Delorme, H.: Magnetic variations on Piton de la Fournaise volcano. Volcanomagnetic signals associated with the November 6 and 30, 1987 eruptions, J. Volcanol. Geotherm. Res., 56, 281-296, 1993. 